\title{
Crosstalk Interconnect Noise Optimization Technique Using Wire Spacing and Sizing for High Speed Integrated Circuits
}

\author{
P. V. Hunagund and A. B. Kalpana
}

\begin{abstract}
Scaling the minimum feature size of VLSI circuits to sub-quarter micron and its clock frequency to $3 \mathrm{GHz}$ has caused crosstalk noise to become a serious problem that degrades the performance and reliability of high speed integrated circuits. This paper presents an efficient method for computing the capacitive crosstalk in sub-quarter micron VLSI circuits. In this paper, we present a complete analytical crosstalk noise model which incorporates all physical properties including victim and aggressor drivers, distributed RC characteristics of interconnects and coupling locations in both victim and aggressor lines. We present closed-form analytical expressions for peak noise and noise width to estimate on-chip crosstalk noise and also shown that crosstalk can be minimized by wire spacing and wire sizing optimization technique. These models are verified for various deep submicron technologies.
\end{abstract}

Index Terms-Aggressor, Coupling, Crosstalk, Interconnect noise, Wire spacing,

\section{INTRODUCTION}

Advancement in the field of very large scale integration (VLSI) have lead to a decrease in device geometries (deep submicron technology), high device densities, high clock rates, and thus small signal transition times. Thus, interconnection lines that were once considered to be electrically isolated can now interfere with each other and have an important impact on system performance and correctness. One such interaction caused by parasitic coupling between wires is known as crosstalk. If not carefully considered during design validation, crosstalk can cause extra signal delay, logic hazards, and even circuit malfunction. Accurate modeling and simulation of interconnect delay due to crosstalk thus becomes increasingly important in the design of high-performance integrated circuits.

The net on which noise is being induced is called the victim net whereas the net that induces this noise is called the aggressor net. Crosstalk noise not only leads to modified delays [2],[3] but also to potential logic malfunctions [4], [5] To be able to deal with the challenges brought by this recently emerging phenomenon, techniques and tools to estimate and avoid crosstalk noise problems should be incorporated into the IC design cycle from the early stages. Any such tool requires fast yet accurate crosstalk noise models both to estimate noise and also to see the effects of

Manuscript received September 19, 2011; revised December 3, 2011

P. V. Hunagund is with the Department of Applied Electronics, Gulbarga University, Gulbarga, India (e-mail:prabhakar.hunagund@gmail.com).

A. B. Kalpana is with the Electronics and Communication Engineering Department, Bangalore Institute of Technology, Bangalore, India (e-mail: abkalpana@gmail.com). various interconnect and driver parameters on noise. Several papers, which propose crosstalk models, can be found in recent literature. In [6], telegraph equations are solved directly to find a set of analytical formulae for peak noise in capacitively coupled bus lines. [7] derives bounds for crosstalk noise using a lumped model but assuming a step input for aggressor driver. The peak noise expression in [7] is extended by [8],[9] to consider a saturated ramp input and a $\pi$ circuit to represent the interconnect. These models fail to represent the distributed nature of the interconnect. In [10], an Elmore delay like peak noise model is obtained for general $\mathrm{RC}$ trees but it assumes an infinite ramp input. This assumption causes the model to significantly overestimate peak noise, especially for small aggressor slews, which is very likely to occur in today's deep submicron designs. Devgan's metric has been improved in [11]. Interconnect crosstalk can be modeled and minimized using different techniques [14],[15].

In this paper, a much improved crosstalk noise model, called the $2 \pi$ - model is discussed. It overcomes major drawbacks of existing models by taking into consideration many key parameters, such as the aggressor slew at the coupling location, the coupling location at the victim net (near-inverter or near-receiver), and the coarse distributed RC characteristics for victim net. It includes simple closed-form expressions to estimate both peak noise and noise width and provides very clear physical meaning for key noise contribution terms. All these characteristics of model $2 \pi$ make it ideal to guide noise-aware layout optimizations explicitly. It is also shown that crosstalk can be minimized by various other optimization techniques [18],[19].

\section{AN IMPROVED 2П CROSSTAlK NOISE MODEL}

\section{A. $2-\pi$ Model and its Analytical Waveform}

For simplicity, we first explain $2-\pi$ model for the case where the victim net is an RC line. We will extend the $2-\pi$ model to a general RC tree in Section 2.3. For a victim net with some aggressor nearby, as shown in Fig. 1(a), let the aggressor voltage pulse at the coupling location be a saturated ramp input with transition time (i.e., slew) being $t_{r}$ and the interconnect length of the victim net before the coupling, at the coupling and after the coupling be $L_{s}, L_{c}$ and $L_{e}$, respectively. The 2- $\pi$ type reduced RC model is generated as shown in Fig. 1(b) to compute the crosstalk noise at the receiver. It is called $2-\pi$ model because the victim net is modeled as two $\pi$-type RC circuits, one before the coupling and one after the coupling. The victim driver is modeled by effective resistance $R_{d}$, Other RC parameters $C_{x}, \mathrm{C}_{1}, R_{s}, C_{2}, R_{e}$, and $C_{L}$ are computed from the geometric information from 
Fig. 1(a) in the following manner. The coupling node (node 2) is set to be the center of the coupling portion of the victim net, i.e., $L_{s}+L_{c} / 2$ from the source. Let the upstream and downstream interconnect resistance capacitance at Node2 be $R_{s} / C_{s}$ and $R_{e} / C_{e}$, respectively. Then capacitance values are set to be $C_{1}=C_{s} / 2, \mathrm{C}_{2}=\left(C_{s}+C_{e}\right) / 2$ and $C_{L}=C_{e} / 2+C 1$. Compared with [12],[13]which only used one lumped RC for the victim net, it is obvious that our $2-\pi$ model can model the coarse distributed RC characteristics. In addition, since we consider only those key parameters, the resulting $2-\pi$ model can be solved analytically.

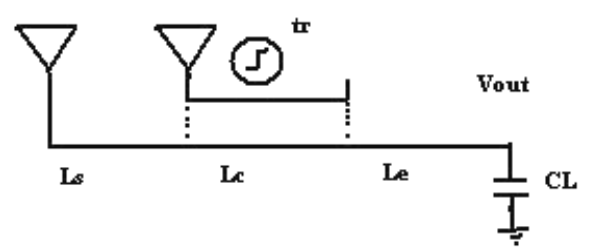

Fig. 1(a). The layout of a victim net and aggressor above it

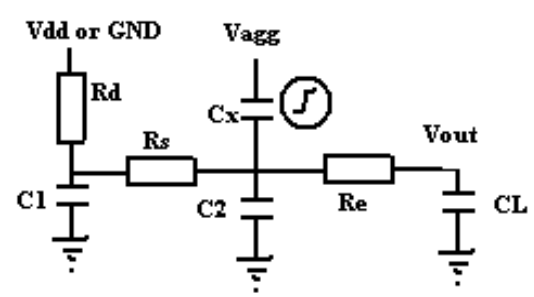

Fig. 1(b). The $2 \pi$ crosstalk noise model.

From Fig. 1(b), we have the impedance at node $1, Z_{1}$ Satisfying the following

$$
\frac{1}{Z_{1}}=\frac{1}{R_{d}}+s C_{1}
$$

Then the s-domain voltage at node 2 by $V_{2}(s)$, then

$$
V_{2}(s)=\frac{Z_{2}}{Z_{2}+\frac{1}{s C_{L}}} \cdot V_{\text {agg }}(s)
$$

The output voltage $V_{\text {out }}$ in the s-domain is

$$
V_{\text {out }}(s)=V_{2}(s) \cdot \frac{\frac{1}{s C_{L}}}{R_{e}+\frac{1}{s C_{L}}}
$$

Substituting $Z_{1}, Z_{2}$ and $V_{2}$ into $V_{\text {out }}(s)$, we have

$$
V_{\text {out }}(s)=\frac{a_{2} s^{2}+a_{1} s}{s^{3}+b_{2} s^{2}+b_{1} s+b_{0}} \cdot V_{a g g}(s)
$$

Writing the transfer function $H(s)$ into the poles/residues form:

$$
H(s)=\mathrm{V}_{\text {out }}(s)=\frac{a_{2} s^{2}+a_{1} s}{s^{3}+b_{2} s^{2}+b_{1} s+b_{0}} \equiv \frac{k_{1}}{s-s_{1}}+\frac{k_{2}}{s-s_{2}}+\frac{k_{3}}{s-s_{3}}
$$

The three poles $s_{1}, s_{2}$ and $s_{3}$ are the three roots of $s^{3}+b_{2} s^{2}+b_{1} s+b=0$, which can be obtained analytically using standard mathematical techniques (details omitted due to page limitation). After each pole/residue pair is obtained, its corresponding time domain function is just $f_{i}(t)=k_{i} e^{S_{i} t} \quad(i=$ $1,2,3)$.

For the aggressor with saturated ramp input and its Laplace transformation is

$$
V_{\text {agg }}(s)=\frac{1-e^{-s t_{r}}}{s^{2} t_{r}}
$$

Then for each pole/residue pair, the s-domain output $V_{\text {out }}(s)=\frac{k_{i}}{s-s_{i}} V_{\text {agg }}(s)$ and its inverse Laplace is

$$
\begin{aligned}
& =\left\{-\frac{k_{i}\left(1+s_{i} t\right)}{s^{2} t_{r}}+\frac{k_{i} e^{s_{i} t}}{s^{2}{ }_{i} t_{r}} \quad 0 \leq \mathrm{t} \leq \mathrm{t}_{\mathrm{r}}\right. \\
& =\left\{-\frac{k_{i} e^{s_{i}\left(t-t_{r}\right)}}{s_{i}{ }^{2} t_{r}}+\frac{k_{i} e^{s_{i} t}}{s^{2}{ }_{i} t_{r}}+\frac{\mathrm{k}_{\mathrm{i}}}{\mathrm{s}_{\mathrm{i}}} \quad \mathrm{t} \geq \mathrm{t}_{\mathrm{r}}\right.
\end{aligned}
$$

Therefore, the final noise voltage waveform is simply the summation of the voltage waveform from each pole/residue pair

$$
v_{\text {out }}(t)=v_{\text {out } 1}(s)+v_{\text {out } 2}(s)+v_{\text {out } 3}(s)
$$

The $2-\pi$ model has been tested extensively and its waveform from (5) can be shown to be almost identical compared to HSPICE simulations.

\section{B. Closed-Form Noise Amplitude and Width}

When although the closed-form noise waveform has been derived in the previous subsection, the solution by itself is still quite complicated. Moreover, it provides little intuition about some key measurements for crosstalk noise, such as noise peak amplitude and noise width, which are very important to guide noise reduction by interconnect optimizations. Simple closed-form expressions for these measurements are highly desired, since they provide more insight about how various interconnect parameters affect the crosstalk noise and to what extent. In this subsection, we will further simplify the original $2-\pi$ model and derive closed-form formulae for noise amplitude and noise width.

Using dominant-pole approximation method in a similar manner like [14], [15], [16], we can simplify (2) into

$$
V_{\text {out }}(s) \approx \frac{a_{1} s}{b_{1} s+b_{0}} \cdot V_{\text {agg }}(s)=\frac{t_{x}\left(1-e^{-s t_{r}}\right)}{s t_{r}\left(s t_{v}+1\right)}
$$

where the coefficient are

$$
\begin{aligned}
& t_{x}=\left(R_{d}+R_{s}\right) C_{x} \\
& t_{v}=\left(R_{d}+R_{s}\right)\left(C_{x}+C_{2}+C_{L}\right)+\left(R_{e} C_{L}+R_{d} C_{1}\right)
\end{aligned}
$$

It is interesting to observe that $t_{x}$ is in fact the RC delay term from the upstream resistance of the coupling element times the coupling capacitance, while $t_{v}$ is the distributed Elmore delay of victim net.

We will further discuss their implications later computing the inverse Laplace transform of (6). We can obtain the following simple time domain waveform

$$
\begin{aligned}
v_{\text {out }} & =\left\{\frac{t_{x}}{t_{r}}\left(1-e^{-\frac{t}{t_{v}}}\right) \quad 0 \leq \mathrm{t} \leq \mathrm{t}_{\mathrm{r}}\right. \\
& =\left\{\frac{t_{x}}{t_{r}}\left(e^{-\frac{\left(t-t_{r}\right)}{t_{v}}}-e^{-\frac{t}{t_{v}}}\right) \mathrm{t}>\mathrm{t}_{\mathrm{r}}\right.
\end{aligned}
$$

It is also interesting to compare with the recent work by [17], where the peak noise with saturated ramp input can be 
written as $v_{\text {max }}^{\prime}=t_{x} /\left(t_{v}+t_{r} / 2\right)$.

Although obtained from a totally different approach, $v^{\prime} \max$ from [17] is indeed a first-order approximation of our $v_{\max }$ in (10), since However, such approximation is only when $\mathrm{t}_{\mathrm{r}}<\mathrm{t}_{\mathrm{v}}$. It will be much off when $\mathrm{t}_{\mathrm{r}}>\mathrm{t}_{\mathrm{v}}$. This explains why $v_{\max }^{\prime}$ in [17] gives twice peak noise.

Peak noise amplitude $V_{\max }$ is not the only metric to characterize noise. Under some circumstance, even the peak noise exceeds certain threshold voltage, a receiver may still be noise immune. This can be characterized by some noise amplitude versus width plots. The noise width is defined as follows.

$$
\begin{aligned}
\frac{t_{x}}{t_{r}}\left(1-e^{-\frac{t_{r}}{t_{v}}}\right) & =\frac{\mathrm{t}_{\mathrm{x}}}{\mathrm{t}_{\mathrm{v}}}\left[1-\frac{1}{2} \frac{t_{r}}{t_{v}}+\ldots . .\right] \\
& \approx \frac{\mathrm{t}_{\mathrm{x}}}{\mathrm{t}_{\mathrm{v}}} \frac{1}{1+\frac{1}{2} \frac{t_{r}}{t_{v}}}=\frac{t_{x}}{t_{v}+\frac{t_{r}}{2}}
\end{aligned}
$$

Definition 1 Noise Width: Given certain threshold voltage level $v_{t}$, the noise width for a noise pulse is defined to be the length of time interval that noise spike voltage $\mathrm{v}$ is larger or equal to $v_{t}$

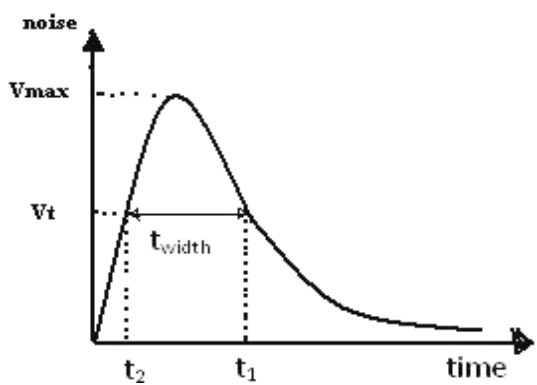

Fig. 2. Illustration of the noise width

From Eq. (9), we can compute $t_{1}$ and $t_{2}$, and thus the noise width

$$
t_{2}-t_{1}=t_{v} \ln \left[\frac{\left(t_{x}-t_{r} v_{t}\right)\left(e^{t_{r} / t_{v}}-1\right)}{t_{r} v_{t}}\right]
$$

In this paper, we set the threshold voltage $v_{t}$ to be half of the peak noise voltage, $v_{t}=v_{\max } / 2$. Then the noise width of (13) is simplified into

$$
t_{\text {width }}=t_{2}-t_{1}=t_{r}+t_{v} \ln \left[\frac{1-e^{-2 t_{r} / t_{v}}}{1-e^{-t_{r} / t_{v}}}\right]
$$

As for the time complexity, since we have the closed-form expressions for the poles, residues, and waveform for each pole/residue pair, the computation time for transfer function and waveform for a given $2-\pi$ model can be done in constant time. To reduce the original circuit to the $2-\pi$ model, we only need a linear traversal (to compute upstream downstream interconnect resistance/capacitance at the coupling node) of the victim net, which can be done in linear time as well as in [12],[18]. It is obviously the lower bound of the computational complexity for any reasonable noise model.

\section{NoISE AVOIDANCE TECHNiQUE}

A general case for two coupled lines is shown in Fig. 3.
Both aggressor and victim lines are divided into 3 regions: interconnect segment before coupling location, coupling location and interconnect segment after coupling location. These regions of aggressor and victim lines are represented by $L_{a l}, L_{c}, L_{a r}, L_{v l}$ and $L_{v r}$ as seen in the Fig. 3 . We propose the linear model shown in Fig.4, to compute crosstalk noise at the receiver of victim net. Victim driver is modeled by effective holding resistance $\mathrm{R}_{\mathrm{h}}$, whereas aggressor driver is modeled by an effective Thevenin model consisting of a saturated ramp voltage source with a slew rate of $t_{r}$ and the Thevennin resistance $R_{t h}$. Other components of our model are computed based on the technology and geometrical information obtained from Fig. 3. Coupling node (node 2 in aggressor net and node 5 in victim net) is defined to be the middle of coupling location for both nets, i.e. $\mathrm{L}_{\mathrm{al}}+\mathrm{L}_{\mathrm{c}} / 2$ away from aggressor driver and $\mathrm{L}_{\mathrm{vl}}+\mathrm{L}_{\mathrm{c}} / 2$ away from the victim driver. For the aggressor net, let the upstream and downstream resistance-capacitance at node 2 be $R_{a 1}-C_{a \mathrm{u}}$ and $R_{a 2}-C_{a d}$ respectively. Then, $C_{a 1}=C_{a u} / 2, C a 2=\left(C_{a u}+C_{a d}\right) / 2$ and $C_{a 3}=$ $C_{a d} / 2+C_{l a}$. Similarly for the victim net, let the upstream and downstream resistance capacitance pair at node 5 be $R_{v 1}-C_{v u}$ and $R_{v 2}-C_{v d}$ respectively. Then, $C_{v 1}=C_{v u} / 2, C_{v 2}=\left(C_{v u}+\right.$ $\left.C_{v d}\right) / 2$ and $C_{v 3}=C_{v d} / 2+C_{\mathrm{l} v}$.

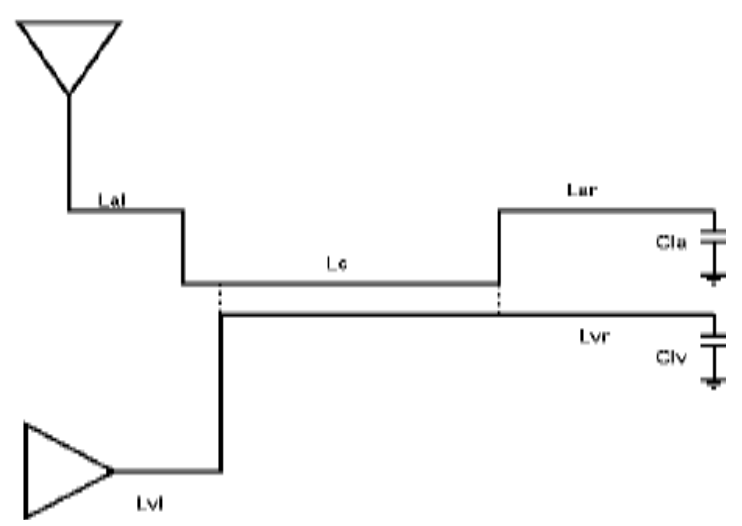

Fig. 3. Linear crosstalk noise model

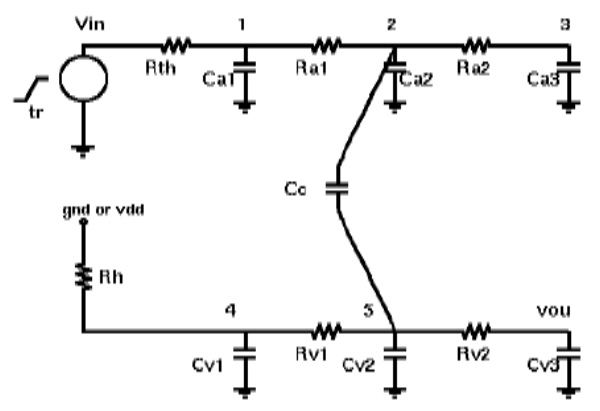

Fig. 4. Linear crosstalk noise model

To simplify the analytical calculation of transfer function $H(s)$ from $V_{\text {in }}$ to $V_{\text {out }}$, we initially decouple the aggressor line from victim line (Fig.5(a)), and compute the transfer function from $V_{\text {in }}$ to $V_{2}$. We then apply $V_{2}(s)$ to the victim line as seen in Fig. 5 (b). This assumption is valid when victim line is not loading aggressor line at node 2 significantly.

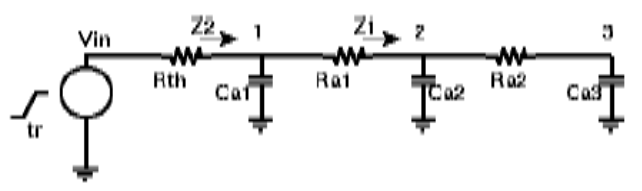

(a) 


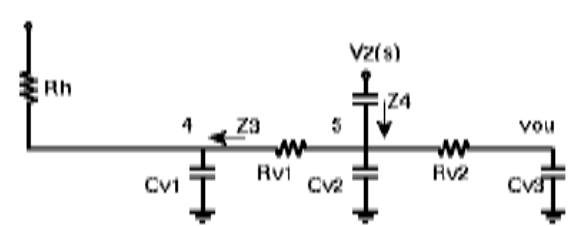

(b)

Fig. 5. Decoupled model to calculate transfer Function.

\section{A. Wire Spacing}

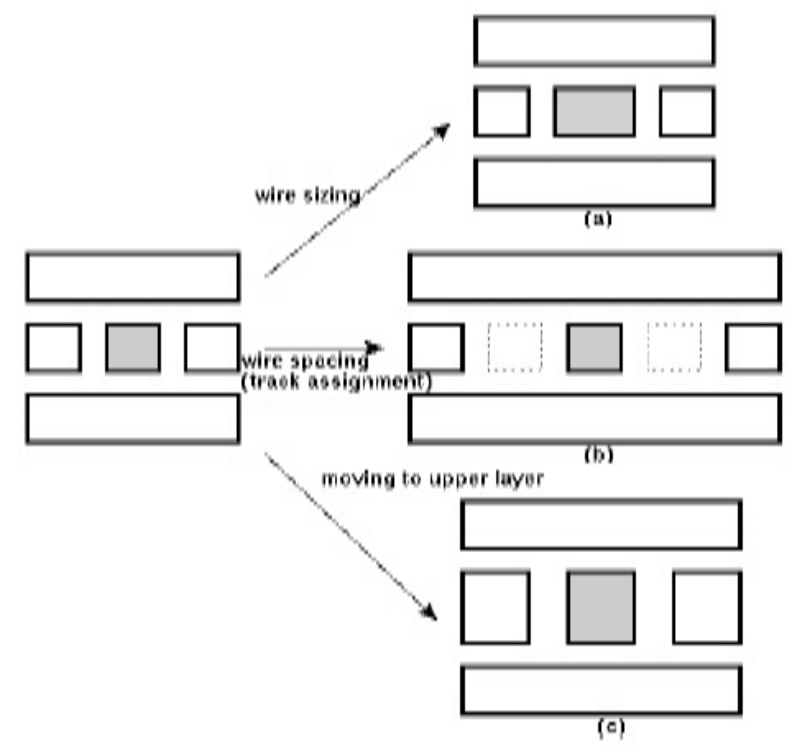

Fig. 6. Cross-section of victim line and surroundings

For a wire of fixed width, its coupling capacitance decreases while its ground capacitance increases, as its spacing to a neighbor wire increases. The decreasing of coupling capacitance is easily explained by the inverse relation between capacitance and distance. Increasing of ground capacitance is due to the fact that as spacing between two wires increases, some of the field lines contributing to coupling capacitance fail reaching the neighbor wire and start contributing to ground capacitance (Fig. 6(b)). From our model,

$$
\begin{aligned}
& \frac{\delta v_{\text {peak }}}{\delta C_{C}}=\left(\frac{R_{h}+R_{v 1}}{t_{r}}\right)\left(1-e^{-t_{r} / t_{v}}\right)-\frac{\left(R_{h}+R_{v 2}\right)^{2} C_{C}}{t_{v}^{2}} e^{-t_{r} / t_{v}} \\
& \frac{\delta v_{\text {peak }}}{\delta C_{v 1}}=\frac{-\left(R_{h}+R_{v 1}\right) C_{C} R_{h}}{t_{v}^{2}} e^{-t_{r} / t_{v}} \\
& \frac{\delta v_{\text {peak }}}{\delta C_{v 2}}=\frac{-\left(R_{h}+R_{v 1}\right)^{2} C_{C}}{t_{v}^{2}} e^{-t_{r} / t_{v}} \\
& \frac{\delta v_{\text {peak }}}{\delta C_{v 3}}=\frac{-\left(R_{h}+R_{v 1}\right) C_{C}\left(R_{h}+R_{v 1}+R_{v 2}\right)}{t_{v}^{2}} e^{-t_{r} / t_{v}} \\
& \frac{\delta v_{\text {peak }}}{\delta C_{a 1}}=\frac{-\left(R_{h}+R_{v 1}\right) C_{C} R_{t h}}{t_{v}^{2}} e^{-t_{r} / t_{v}} \\
& \frac{\delta v_{\text {peak }}}{\delta C_{a 2}}=\frac{-\left(R_{h}+R_{v 1}\right) C_{C}\left(2 R_{a 1}+R_{t h}\right)}{t_{v}^{2}} e^{-t_{r} / t_{v}} \\
& \frac{\delta v_{\text {peak }}}{\delta C_{a 3}}=\frac{-\left(R_{h}+R_{v 1}\right) C_{C}\left(2 R_{a 1}+2 R_{a 2}+R_{t h}\right)}{t_{v}^{2}} e^{-t_{r} / t_{v}}
\end{aligned}
$$

From Eq.(15), $\delta v_{\text {peak }} / \delta C_{C}$ is positive but diminishes when $t_{v}>t_{r}$ in which case reduction in coupling capacitance doesn't help peak noise reduction. Eqn's $(16,17,18,19,20$, 21 ) show that an increase in the ground caps of both victim and aggressor lines help reduce noise on the victim net. Their relative effectiveness's are as follows.

$$
\begin{aligned}
& \frac{\delta v_{\text {peak }}}{\delta C_{v 3}}<\frac{\delta v_{\text {peak }}}{\delta C_{v 2}}<\frac{\delta v_{\text {peak }}}{\delta C_{v 1}}<0 \\
& \frac{\delta v_{\text {peak }}}{\delta C_{a 3}}<\frac{\delta v_{\text {peak }}}{\delta C_{a 2}}<\frac{\delta v_{\text {peak }}}{\delta C_{a 1}}<0
\end{aligned}
$$

As can be seen from (22 - 23), for the same amount of increase in ground capacitance, peak noise reduction is most effected from near sink capacitances in both victim and aggressor lines.

\section{B. Wire Sizing}

As a wire's width is increased, its resistance decreases and its ground capacitance increases (Fig. 6(a)). If we look at how noise peak is affected by changes in interconnect resistances, we get the following sensitivities from our model.

$$
\begin{aligned}
& \frac{\delta v_{\text {peak }}}{\delta R_{v 1}}=\frac{C_{C}}{t_{r}}\left(1-e^{-t_{r} / t_{v}}\right)- \\
& \left(R_{h}+R_{v 1}\right) \frac{C_{C}\left(C_{C}+C_{v 2}+C_{v 3}\right)}{t_{v}^{2}} e^{-t_{r} / t_{v}} \\
& \frac{\delta v_{\text {peak }}}{\delta R_{v 2}}=\frac{-\left(R_{h}+R_{v 1}\right) C_{C} C_{v 3}}{t_{v}^{2}} e^{-t_{r} / t_{v}} \\
& \frac{\delta v_{\text {peak }}}{\delta R_{a 1}}=\frac{-2\left(R_{h}+R_{v 1}\right) C_{C}\left(C_{a 2}+C_{a 3}\right)}{t_{v}^{2}} e^{-t_{r} / t_{v}} \\
& \frac{\delta v_{\text {peak }}}{\delta R_{a 2}}=\frac{-2\left(R_{h}+R_{v 1}\right) C_{C} C_{a 3}}{t_{v}^{2}} e^{-t_{r} / t_{v}}
\end{aligned}
$$

Equation (24) shows that the effect of $R_{v 1}$ on noise reduction is very similar to that of $R_{h}$. On the other hand, from Equations $(25,26,27)$, the effects of $R_{v 2}, R_{a 1}$ and $R_{a 2}$ are opposite.

Peak noise increases as these resistances are decreased. As a result, when a victim wire's width is increased, the change in peak noise depends on Eqn's (16, 17, 18, 25, 26). Eqn's (25 and 26) show the importance of coupling location on how effective wire sizing will be. If the coupling location is close to victim driver, Eq.(26) will be more effective than Eq. (25) and thus effect of wire sizing on noise reduction will diminish. Wire sizing will be most effective when coupling location is close to victim receiver.

On the other hand, the effect of increasing an aggressor wire's width depends on relative magnitudes of $\delta v_{\text {peak }} / \delta C_{a i}$ and $\delta v_{\text {peak }} / \delta R_{a i}$. By looking at Eqn's $(19,20,21,26,27)$, it can be seen that the capacitance sensitivities are greater in magnitude than resistance sensitivities. Thus if $R_{a i}$ decrease as much as $C_{a i}$ increase as a result of width increase, this will help reduce noise on the victim receiver input. For validation interconnect parameters are considered from $32 \mathrm{~nm}$ and $55 \mathrm{~nm}$ technologies. Output voltages are observed for normal driver size and also by increasing driver size as shown in Table $1,2,3 \& 4$.It is observed that crosstalk can be minimized by interconnect optimization. 


\section{CMOS 32nm Technology}

TABLE I: NORMAL WIRE SIZE WITH PROPAGATION DELAY: 50NSEC

\begin{tabular}{|l|c|c|c|c|}
\hline \multicolumn{1}{|c|}{ Net } & $\mathrm{R}_{\mathrm{s} 1}(\mathrm{~K} \Omega)$ & $\mathrm{C}_{\mathrm{c} 1}(\mathrm{pF})$ & $\mathrm{C}_{\mathrm{sub} 1}(\mathrm{e}-17 \mathrm{~F})$ & Output $(\mathrm{V})$ \\
\hline Aggressor & 1.12 & 2.97 & 1.2 & \multirow{2}{*}{4} \\
\cline { 1 - 4 } Victim & 1.11 & 2.97 & 1.2 & \multirow{2}{*}{4.7} \\
\cline { 1 - 4 } Net & $\mathrm{C}_{\mathrm{c} 2}(\mathrm{pF})$ & $\mathrm{C}_{\mathrm{sub} 2}(\mathrm{e}-19 \mathrm{~F})$ & $\mathrm{R}_{\mathrm{s} 2}(\mathrm{~K} \Omega)$ & \\
\hline Aggressor & 1.1 & 3.73 & 6.42 & \\
\cline { 1 - 4 } Victim & 1.1 & 3.86 & 6.56 & \\
\hline
\end{tabular}

TABLE II: OPTIMIZED WIRE SPACE AND WIRE WIDTH

\begin{tabular}{|c|c|c|c|c|}
\hline Net & $\mathrm{R}_{\mathrm{s} 1}(\mathrm{~K} \Omega)$ & $\mathrm{C}_{\mathrm{c} 1}(\mathrm{e}-19 \mathrm{~F})$ & $\mathrm{C}_{\text {sub1 }}(\mathrm{e}-17 \mathrm{~F})$ & Output $(\mathrm{V})$ \\
\hline Aggressor & 0.01 & 3.76 & 1.2 & \multirow{2}{*}{4} \\
\cline { 1 - 4 } Victim & 0.01 & 3.76 & 1.1 & \multirow{2}{*}{4.96} \\
\cline { 1 - 4 } Net & $\mathrm{C}_{\mathrm{c} 2}(\mathrm{e}-19 \mathrm{~F})$ & $\mathrm{C}_{\mathrm{sub} 2}(\mathrm{e}-19 \mathrm{~F})$ & $\mathrm{R}_{\mathrm{s} 2}(\mathrm{~K} \Omega)$ & \\
\hline Aggressor & 9.46 & 3.73 & 3.48 & \\
\cline { 1 - 4 } Victim & 9.46 & 3.86 & 3.40 & \\
\hline
\end{tabular}

\section{CMOS 55nm Technology}

TABLE III: NORMAL WIRE SIZE WITH PROPAGATION DELAY: 45NS

\begin{tabular}{|c|c|c|c|c|}
\hline Net & $\mathrm{R}_{\mathrm{s} 1}(\Omega)$ & $\mathrm{C}_{\mathrm{c} 1}(\mathrm{e}-16 \mathrm{~F})$ & $\mathrm{C}_{\text {sub1 }}(\mathrm{e}-15 \mathrm{~F})$ & Output $(\mathrm{V})$ \\
\hline Aggressor & 45 & 1.299 & 8.464 & \\
\cline { 1 - 4 } Victim & 37 & 1.299 & 8.464 & \multirow{2}{*}{4.5} \\
\cline { 1 - 4 } Net & $\mathrm{C}_{\mathrm{c} 2}(\mathrm{e}-19 \mathrm{~F})$ & $\mathrm{C}_{\mathrm{sub} 2}(\mathrm{e}-15 \mathrm{~F})$ & $\mathrm{R}_{\mathrm{s} 2}(\Omega)$ & \\
\cline { 1 - 4 } Aggressor & 1.28 & 8.4 & 307 & \\
\cline { 1 - 4 } Victim & 1.27 & 8.4 & 316 & \\
\hline
\end{tabular}

TABLE IV: OPTIMIZED WIRE SPACE AND WIRE WIDTH

\begin{tabular}{|c|c|c|c|c|}
\hline Net & $\mathrm{R}_{\mathrm{s} 1}(\Omega)$ & $\mathrm{C}_{\mathrm{c} 1}(\mathrm{e}-18 \mathrm{~F})$ & $\mathrm{C}_{\text {sub1 }}(\mathrm{e}-14 \mathrm{~F})$ & Output $(\mathrm{V})$ \\
\cline { 1 - 4 } Aggressor & 0.001 & 5.76 & 1.2 & \multirow{2}{*}{4.96} \\
\cline { 1 - 4 } Victim & 0.001 & 5.76 & 1.2 & \\
\cline { 1 - 4 } Net & $\mathrm{C}_{\mathrm{c} 2}(\mathrm{e}-19 \mathrm{~F})$ & $\mathrm{C}_{\mathrm{sub} 2}(\mathrm{e}-14 \mathrm{~F})$ & $\mathrm{R}_{\mathrm{s} 2}(\Omega)$ & \\
\cline { 1 - 4 } Aggressor & 1.32 & 1.2 & 0.001 & \\
\cline { 1 - 4 } Victim & 1.32 & 1.12 & 0.001 & \\
\hline
\end{tabular}

\section{CONCLUSION}

In this paper, we presented a $2 \pi$ crosstalk noise model which incorporates all victim and aggressor driver/ interconnect physical parameters including coupling locations on both victim and aggressor nets. We derived analytical expressions for the important metrics of crosstalk noise height and width using this model. Crosstalk noise minimization technique using on-chip wire spacing and sizing are also developed and validated for deep submicron technologies. Output voltage is observed for normal wire size as well as for optimized wire size/spacing and shown that crosstalk can be minimized by interconnects optimization.

\section{REFERENCES}

[1] S. I. Association, "The international technology roadmap for semiconductors", 1999.

[2] J. Cong, D. Zhingang, and P. V. Srinivas, "Improved crosstalk modeling for noise constrained interconnect optimization", In Proceedings of ASP/DAC Asia South Pasific Design Automation Conference, pp. 373-378, 2001.

[3] P. D. Gross, R. Arunachalam, K. Rajagopal and L. T. Pileggi, "Determination of worst-case aggressor alignment for delay calculation", In Proceedings of the IEEE International Conference on Computer-Aided Design, ICCAD-98, 1998.

[4] S. Sirichotiyakul, D. Blaauw, C. Oh, R. Levy, V. Zolotov and J. Zuo. Driver modeling alignment for worst-case delaynoise. In Proceedings of Design Automation Conference DAC, pp. 720-725, June 2001.

[5] S. Alwar, D. Blaauw, A. Dasgupta, A. Grinshpon, R. Levy, C. Oh, B. Orshav, S. Sirichotiyakul and V. Zolotov. Clarinet: A noise analysis tool for deep submicron design. In Proceedings of Design Automation Conference DAC, pp. 233-238, June 2000.
[6] K. L. Shepard and V. Narayanan, "Noise in deep submicron digital design", In Proceedings of ICCAD-96 Intl. Conferenceon Computer Aided Design, pp. 524-531, Nov. 1996.

[7] T. Sakurai., "Closed-form expression for interconnect delay, coupling, and crosstalk in VLSIs", IEEE Transactions on Electron Devices, 40:118-124, 1993

[8] A. Vittal and M. Marek-Sadowska, "Crosstalk reduction for VLSI", IEEE Transactions on Computer Aided Design, 16:290-298, March 1997.

[9] A. Vittal, L. H. Chen, M. Marek-Sadowska, K. P. Wang and S. Yang. "Crosstalk in VLSI interconnections", IEEE Transactions on Computer Aided Design, 18:1817-1824, Dec. 1999.

[10] A. B. Kahng, S. Muddu, and D. Vidhani. Noise and delay uncertainty studies for coupled rc interconnects. In Proceedings of ASIC/SOC Conference, pages 3-8, 1999.

[11] A. Devgan, "Efficient coupled noise estimation for on-chip interconnects", In Proceedings of the IEEE International Conference on Computer-Aided Design, ICCAD-97, pp. 147-153, 1997.

[12] M. Kuhlmann and S. S. Sapatnekar. Exact and efficient crosstalk estimation. IEEE Transactions on Computer Aided Design, 20(7):858-866, July 2001.

[13] A. Vittal and M. Marek-Sadowska, "Crosstalk reduction for VLSI," IEEE Trans. on Computer-Aided Design of Integrated Circuits and Systems, Vol. 16, pp.290-98, 1997.

[14] S. Nakagawa, D. M. Sylvester, J. McBnde, and S. Y. Oh, "On-chip cross talk noise model for deep submicrometer ulsi interconnect," Hewlett-Packard Journal, vol. 49, pp. 39-45, Aug. 1998.

[15] A.B. Kahng, S. Muddu, and D. Vidhani, "Noise and delay uncertainty studies for coupled rc interconnects," in IEEE International ASIC/SOC Conference, pp. 3-8,1999

[16] M. Kuhlmann, S. Sapamekar, and K. Parhi, "Efficient crosstalk estimation," in Proc. IEEE International Conference on Computer Design, pp. 266-272, 1999.

[17] E. Acar, A. Odabasioglu, M. Celik, and L. Pileggi, "S2p:a stable 2-pole RC delay and coupling noise metric IC interconnects," in Proceedings 9th Great Lakes Symposium on VLSI, pp. 63, 1999.

[18] A. Vittal, L. Chen, M. Marek-Sadowska, K.-P. Wang and S. Yang, "Crosstalk in VLSI Interconnections" IEEE Trans. on Computer-Aided Design of Integrated Circuits and System, Vol. 18, No. 2.

[19] A. Devgan, "Efficient coupled noise estimation for on-chip interconnects," in Proc. Int. Con5 on Computer Aided Design, pp. $147-153,1997$

[20] Xiaopeng Ji, Long Ge, Xiaodong Han, Zhiquan Wang, “Crosstalk Noise Analysis for Distributed Parameter High-Speed Interconnect Lines Based on the Transfer Function”, 978-1-4244-1734-6/08/\$25.00/ 2008 IEEE

[21] Md. Sajjad Rahaman and Masud H. Chowdhury "Crosstalk Avoidance and Error-Correction Coding for Coupled RLC Interconnects", 978-1-4244-3828-0/09/\$25.00 C2009 IEEE

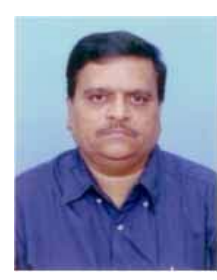

P. V. Hunagund received his M.Sc and Ph.D from the Department of Applied Electronics, Gulbarga University, Gulbarga, in the year 1982 and 1992 respectively. $\mathrm{He}$ is the Senior Professor of Applied Electronics Department, Gulbarga University, Gulbarga, INDIA.

$\mathrm{He}$ has more than 50 research publications in national and international reputed journals, more than 155 research publications in international symposium/Conference and more than 100 research publications in national symposium/Conference. He presented many papers in India \& abroad. He has guided many Ph.D and M.Phil students. He has completed three major research projects funded by A.I.C.T.E. and D.S.T. New Delhi. At present he is the Coordinator of the Non-SAP project funded by UGC, New Delhi.

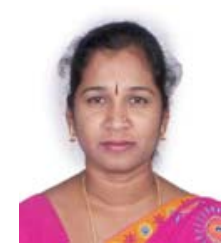

A. B. Kalpana received B.E, Degree from, S.J.C.I.T, Bangalore University, In 1995, M.E, Degree from U.V.C.E, Bangalore University, Bangalore, India in 2001, pursuing Ph.D in the Department of Applied Electronics, Gulbarga University, Gulbarga, INDIA, currently she is working as Assistant Professor in the Department of Electronics and communication, Bangalore Institute of Technology, Bangalore, INDIA, her research interests include Analysis and Design of VLSI circuits and Power Electronics. 